\title{
A Relationship between Movement Time and Traveled Distances in Forward/Backward direction Cooperative Task
}

\author{
Ahmad Faizal S., Ryojun Ikeura, Soichiro Hayakawa, and Hideki Sawai \\ Division of Mechanical Engineering, Graduate School of Engineering, Mie University, Tsu, 514-8507, \\ Japan \\ faizal@ss.mach.mie-u.ac.jp
}

\begin{abstract}
Keywords: Human-human system, Cooperative object transfer, Human-robot system, Movement time, Minimum Jerk Model
\end{abstract}

\begin{abstract}
The research main objective was to generate a smooth cooperative rigid object transfer by a human hand and a robot manipulator mimicking the same task performs by two humans. In achieving such objective, the relationship between movement time and traveled distances of the cooperative task performed by two humans were investigated. A motion capture system was utilized to record the movement of two subjects namely, Leader and Follower during the cooperative transfer of an object using their right hands in a seated position. The cooperative task was performed in forward/backward directions relative to the Leader. The Leader informed the Follower on the onset and the terminating position prior to the execution of the cooperative task. Both subjects moved the object towards the target together. The results show a linear relationship between movement time and traveled distances. Both subjects were found to have similar relationship for the cooperative task in the same direction. In forward/backward cooperative task, the direction is not significant in influencing the relationship between movement time and traveled distances. The relationship will be tested with robotic motion in the next phase of the research.
\end{abstract}

\section{Introduction}

Human-robot cooperation is an emerging field that broadens the research opportunity in robotic technology. Arm-manipulator coordination in carrying an object simultaneously from one position to another is an example of various researches under human-robot cooperation field. A manipulator motion mimicking the smoothness and naturalness of human arm motion influences the acceptance of robotics technology for effective collaboration.

Various researchers have studied on the generation of a smooth arm-manipulator cooperative motion. Glasauer S. et al studied a smooth, natural and efficient hand over task in human-human and human-robot system [1]. In human-robot system, a robotic motion based on the minimum jerk model provided better end-point motion estimation to the human receiver. Maeda et al investigated a control method for robot in human-robot cooperative manipulation system [2]. In their study, the robot did not know the desired location of the human partner. Thus, the human partner arm motion was estimated using the minimum jerk model. Finally, the estimated arm position was used to determine the desired position of the end effector of the manipulator in virtual compliance control. However, none of the literatures studied on a cooperative task with predefined target location and movement onset e.g. arm-manipulator cooperative motion for part assembly task and etc.

The main objective of this research was to generate a smooth and natural cooperative rigid body transfer by a human hand and a robot manipulator mimicking the same smoothness and naturalness of cooperative task performed by two humans. The robot acts as a Follower and the human as a Leader mimicking the role of Follower and Leader in human-human system. In a real cooperative task involving two humans, one of the agents may not know the desired location and movement onset of the other agent. Thus, we limit our study to a skilled cooperative task with predefined target location and movement onset e.g. human-human cooperation for part assembly task and etc. The predefined target location and movement onset mean that one subject informed about the target location and instructed the other subject on the task initiation prior to the task execution, respectively. A skilled cooperative task means that it had been executed frequently and both subjects familiar with each other 
motion. Within these limits, the requirement to feedback one subject motion to the other during the cooperative task was omitted [3]. Therefore, in generating a smooth cooperative object transfer in human-robot system mimicking the same smoothness in human-human system, the motion of the human Leader is not required to be feedback to the robot Follower. The robot could be programmed based on a smooth trajectory of the human Follower in human-human cooperative system to generate a smooth cooperative task. Moreover, the smooth trajectory agreed with the trajectory generated using the Minimum Jerk Model (MJM) [4]. Thus, the trajectory of the robot Follower will be based on the trajectory of the MJM. In using the MJM to generate a smooth trajectory for the robot Follower mimicking the trajectory of human Follower (human-human system), the relationship between movement time and traveled distances of human Follower is required.

Therefore, in this article, the relationship between cooperative task movement time and traveled distances was investigated. It was mentioned that hand movement towards target consisted of two motion phases, the transfer phase from start to target position and precise positioning phase of object at target area [5]. This research was carried out to resemble mainly the transfer phase (precise positioning phase was excluded) of actual part assembly in industries. Furthermore, the research was targeted to be applied at an environment requiring changes of fixed human partner (e.g. shift working industries, 3 workers, in each shift only one worker act as a Leader) working with a single robot (robot act as a Follower). Thus, a single robot could be programmed with several human hand motion characteristics (referring to the relationship between movement time and travel distances) and at a time, only a characteristic that suit to a particular human partner is selected and use for executing the cooperative task. The selection of each person characteristic must be made available prior to the task execution. The article discussed on the skilled task, where they practised several times before the task was executed and the feedback requirement of each other motion was eliminated. Without the feedback requirement, sometimes the Follower may move earlier and faster than the Leader during the task which indicates the insignificant of the role as Leader and Follower after the task initiation [3]. Thus, the role of Leader and Follower is actually limited on the task onset (when Leader instructs Follower to move the object) not during the transfer phase. Since the article focused mainly on the transfer phase, a detail discussion of the groups involved in the change of a role of Leader and Follower over the same subject was omitted.

\section{The Minimum Jerk Model}

The MJM adapts the optimal control methodology and had been proven to predict the kinematics aspect of smooth human arm motion which includes the arm position, velocity, acceleration and jerk. Jerk is defined as the change of rate of acceleration and thus, it is a third temporal derivative of position. Assuming that $x$ and $y$ are the time varying hand positions in the Cartesian coordinate system, the magnitude of the jerk to be minimized can be shown as,

$$
\text { Jerk }=\sqrt{\left(\frac{d^{3} x}{d t^{3}}\right)^{2}+\left(\frac{d^{3} y}{d t^{3}}\right)^{2}}
$$

In moving a human hand from an initial to a final position in a given time $t$, the cost function $C$ to be

$$
\mathrm{C}=\frac{1}{2} \int_{0}^{t_{f}}\left[\left(\frac{d^{3} x}{d t^{3}}\right)^{2}+\left(\frac{d^{3} y}{d t^{3}}\right)^{2}\right] d t
$$

where, $t_{f}(\mathrm{sec})$ is the time at termination of movement. In our experiment, a one dimensional movement is considered; therefore the above minimum jerk formula can be simplified as,

$$
\mathrm{C}=\frac{1}{2} \int_{0}^{t} f\left[\left(\frac{d^{3} x}{d t^{3}}\right)^{2}\right] d t
$$

Although the MJM is used for a single human operator, in our case, we assume that for a smooth cooperative motion, one subject will follow another subject movement while maintaining his/her own jerk at minimum level. Based on this concept, the MJM is used in our experiment to evaluate the smoothness of human-human cooperative tasks quantitatively. Solving equation (3), the following one dimensional minimum jerk trajectory is obtained. 


$$
x(t)=x_{i}+\left(x_{f}-x_{i}\right)\left(10\left(\frac{t}{-}\right)-15\left(\frac{t}{-}\right)+6\left(\frac{t}{6}\right)\right)
$$

where $x_{i}, x_{f}$ and $t_{f}$ are initial position, final position and movement time from initial to final position, respectively. The $\left(x_{f}-x_{i}\right)$ component indicates the traveled distances during the cooperative motion. Thus, obtaining the movement time, $t_{f}(\mathrm{~s})$ and traveled distances, $D(\mathrm{~m})$ relationship in the cooperative motion in human-human system will enable us to implement the minimum jerk model in human-robot cooperative system.

\section{Experimental Method and Equipments}

Experimental Devices. The experimental equipment consisted of position measurement system (Optotrack Certus 3020 camera, System Control Unit and Personal Computer), two monitor screens, two height-adjustable chairs and an experimental object as shown in Fig. 1. Fig. 2 shows the experimental object dimensions and configuration. The dimensions of the experimental object are $18 \mathrm{~cm}(\mathrm{~W}) \times 6 \mathrm{~cm}(\mathrm{H}) \times 46 \mathrm{~cm}(\mathrm{~L})$ and weighted about $3 \mathrm{~kg}$. It is equipped with small infrared light emitting diode (IR LED) markers on its sides. When both subjects moved the experimental objects in any directions, the $3 \mathrm{D}$ camera in the position measurement system detected signals from the diode marker and sent it to the main computer. The signals (position data) were processed and converted to an image mimics the experimental object motion (rotational and translational) in real time and the image was shown on the screen in front of both subjects. The image size is $10 \mathrm{~mm}(\mathrm{~W}) \times 100 \mathrm{~mm}(\mathrm{~L})$. Position data were recorded into the computer at every $10 \mathrm{~ms}$ of sampling interval. Fig. 3 shows the image, start and target position on the screen monitor. It was very crucial for both subjects to position the experimental object's image to a precise target with smoothness and naturalness of hand motion [3]. Therefore, the target was drawn as two parallel lines and if its centerline coincides with rectangular image's centerline, it provides $3 \mathrm{~mm}$ gaps to tolerate the image positioning. The targets increased the effectiveness of the cooperative task because it helped Follower to know the task's termination position on the screen. The targets were positioned in four types of distances in each direction. The distance was set at 35, 70, 100 and 200mm as shown in Fig. 3.

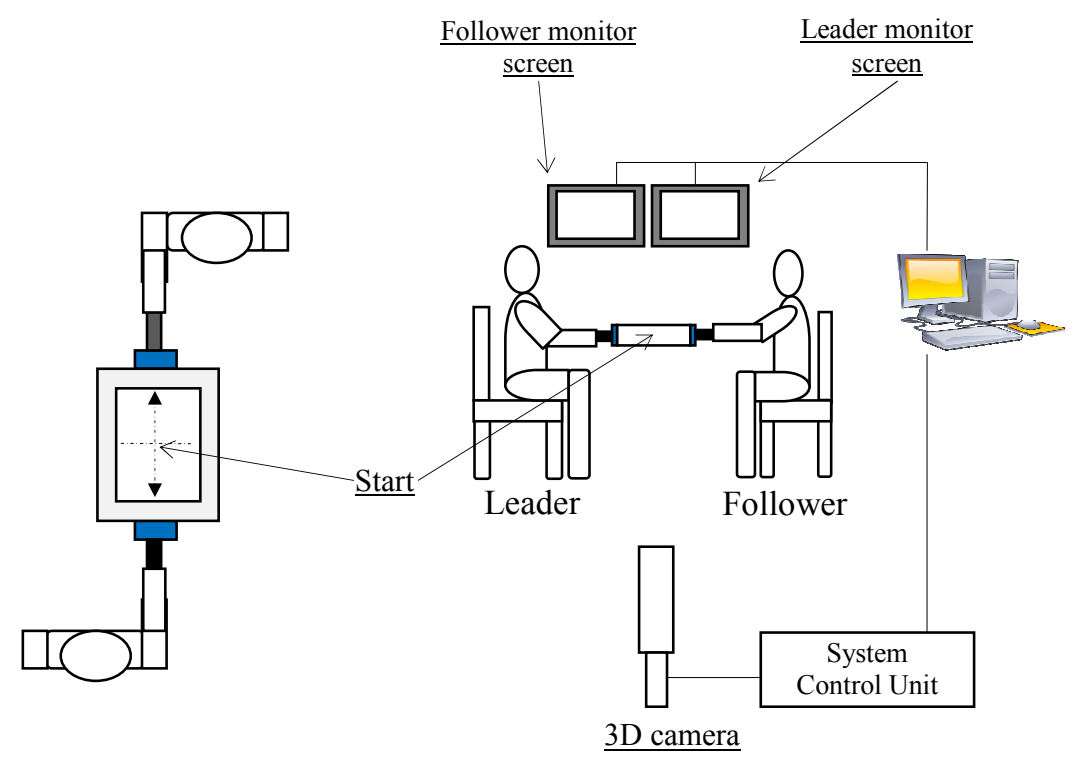

Fig. 1. Layout of experimental devices for forward/backward cooperative task 

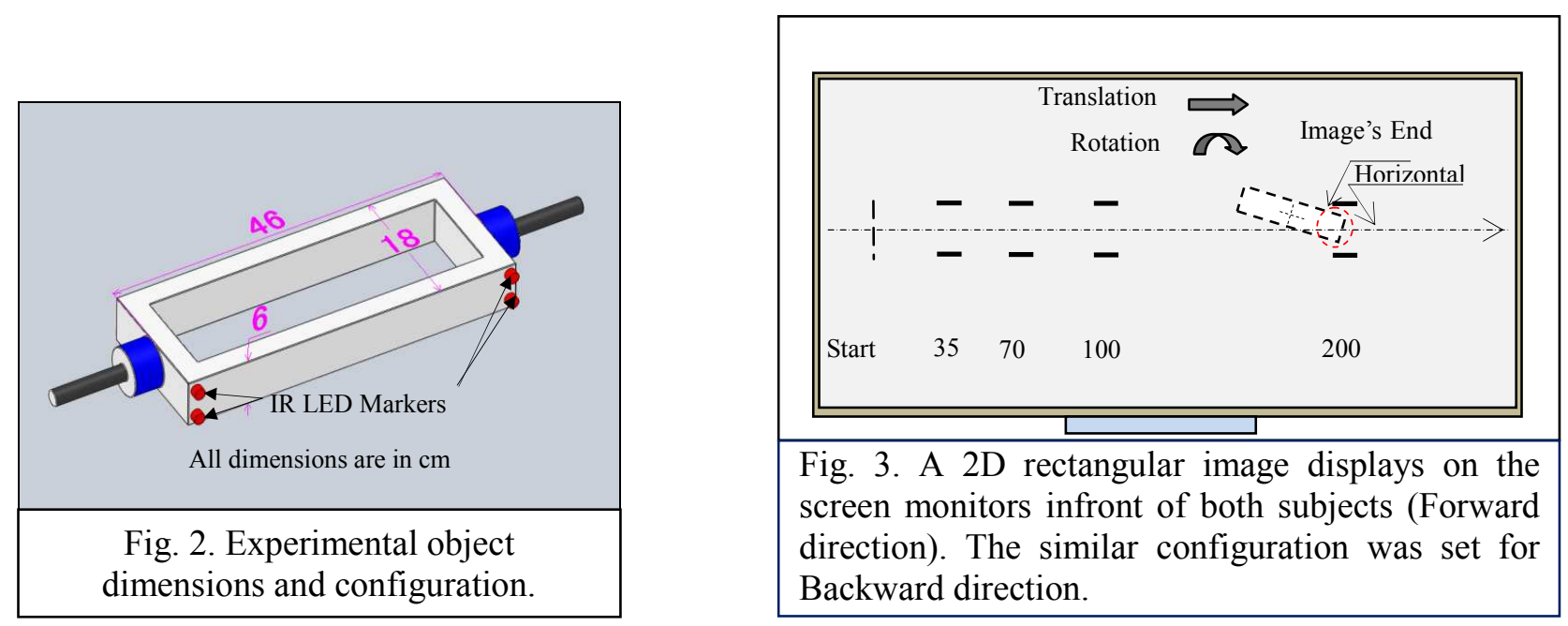

Experimental Procedure. The experiment was design to understand the relationship between movement time and traveled distances of a smooth cooperative task where both subjects knew the target and on the onset of the task. Three engineering students had been selected as experimental subjects which aged between 20 to 40 years old, physically and mentally health. Subjects did not report any sensory, neurological, muscular, cutaneous or impaired related problems. All of them had no experiences in executing the current experiment. All subjects were provided with informed consents and instructions on the experiment. In executing cooperative object transfer by two humans, one person acts as a Leader and the other as a Follower. Thus, alternating three subjects (A, B, and C) as Leader and Follower in each group, six groups $(\mathrm{Ab}, \mathrm{Ac}, \mathrm{Ba}, \mathrm{Bc}, \mathrm{Ca}$, and $\mathrm{Cb})$ were formed. The capital letter and the small letter indicate the Leader and the Follower, respectively. During the experiment, one subject known as host works at the computer (start up software, recording and etc) and another two subjects work as Leader and Follower at the experimental object area. The Leader and Follower sat on their respective chair. The distance between the chairs was fixed for each group and it was decided so that all subjects could move smoothly from the start position to the farthest target on the screen in all cooperative task directions (leftward/rightward, upward/downward and forward/backward). Host started up the software and an image resembling the real experimental object appeared on the screen in front of both subjects. Host instructed both subjects to hold the experimental object and match the image resembling the experimental object on the starting position. The starting position on the screen was similar to the middle point located between Leader and Follower. Mimicking the real cooperative task, the Leader initiated, decided and informed the direction and target position of the task to the Follower. A verbal signal was given to the Follower by the Leader to indicate cooperative task initiation. Once started, the Follower cooperated with the Leader in bringing the experimental object from the start point to the target position together. The Follower was not required to observed and follow the Leader motion during the task. Once reached the target, both subjects returned the experimental object to the starting position. Both of them were required to move the experimental object in upward/downward, leftward/rightward and forward/backward) direction relative to the Leader. The 3D camera recorded their cooperative motion and position data were saved in the computer. As mention in introductory section, the research focused on the transfer task during assembly and not on the precise positioning. Thus, the targets were merely provided as a guideline to terminate a smooth cooperative transfer motion. Both subjects were allowed to terminate the cooperative task as they approached near to the target area (only data with half of the image reached the target was considered). The subjects were required to practice by moving the object for several times before proceeding with the experiments. The practice was very important so that the subject could adjust to the change of the experimental conditions and familiarize with each other motion (eliminate the feedback of the Leader motion to the Follower). Furthermore, it resembled a skilled motion (as it is done daily) performed by the industrial workers. The experiments 
were conducted with 10 repetitions for each direction and distance. Only smooth motions were selected in each distance and direction. The selection was based on the calculated minimum jerk value, where value less than 500 (normalized jerk) was considered as a smooth cooperative motion. Statistical analyses were performed using the Statistical Packages for the Social Sciences (SPSS). Differences between the levels of each variable were detected using ANOVA. A significant level of 0.01 was used for all the statistical tests.

\section{Results}

The scatter plots of cooperative movement time, $t(\mathrm{~s})$ versus traveled distances, $D(\mathrm{~m})$ is shown in Fig. 4. Group name was indicated at the top of each plot, where the capital letter refers to the Leader and the small letter refers to the Follower. The symbol within each plot was classified based on subjects (Leader and Follower) and directions.

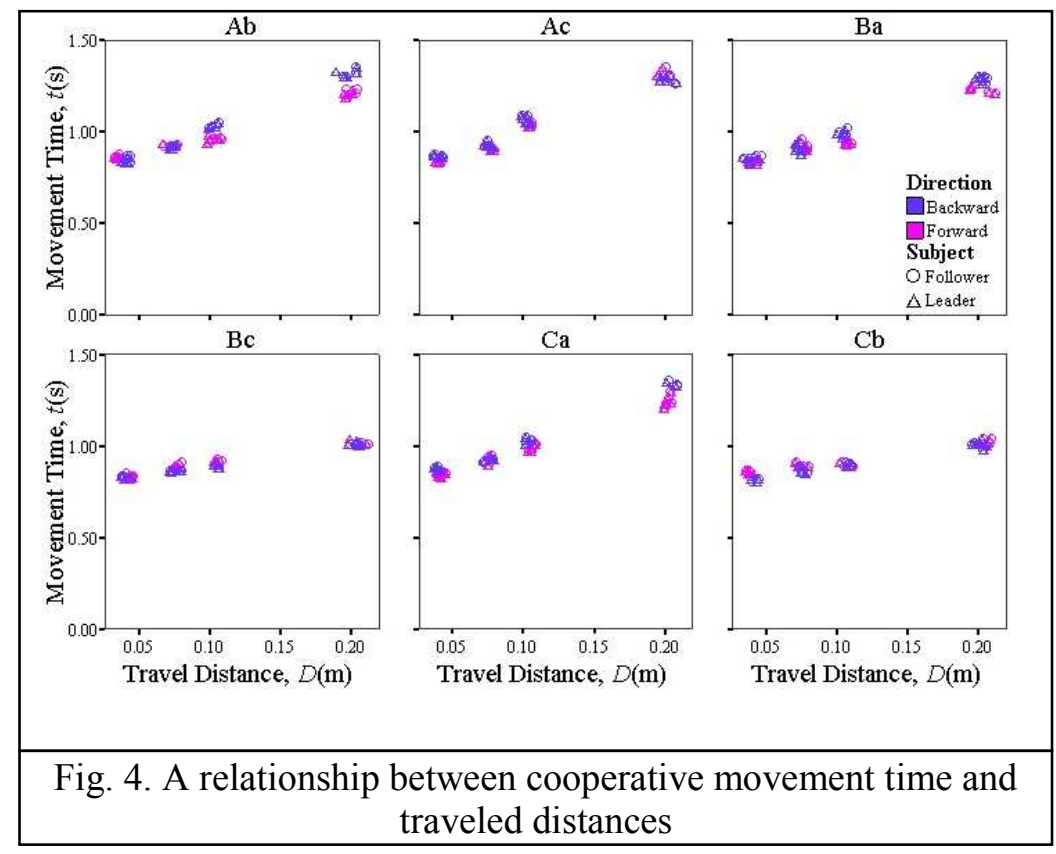

The movement time was significantly related to the traveled distances, $p<0.01$. The results indicated that for a smooth task involving two humans carrying a rigid object together from one position to a certain target; an increased in the target distance resulted in a linear increment of the time to perform the task. All positive correlation coefficients were shown in the following table1.

Table 1 Pearson correlation coefficients

\begin{tabular}{lcccccc}
\hline Groups & $\mathrm{Ab}$ & $\mathrm{Ac}$ & $\mathrm{Ba}$ & $\mathrm{Bc}$ & $\mathrm{Ca}$ & $\mathrm{Cb}$ \\
\hline Coefficients & 0.972 & 0.981 & 0.975 & 0.901 & 0.983 & 0.941 \\
\hline
\end{tabular}

The following equation represents the general equation for a linear relationship between cooperative movement time, $t(\mathrm{~s})$ and travel distances, $D(\mathrm{~m})$,

$$
t=m^{*} D+t_{0}
$$

where $m$ is the gradient of a line representing the linear relationship, and $t_{0}$ is the starting time. Eq. 5 is true for the cooperative task with a distance equal and more than $35 \mathrm{~mm}$ as conducted in this experiment. In human-robot cooperative system, the robot will act as a Follower and its manipulator is aimed to mimic human hand motion. Thus, in this article, the movement time and traveled distances relationship of the Follower in human-human system was thoroughly investigated and to be utilized in generating manipulator motion in human-robot system. Fig. 5 shows the scatter plots of the movement time and traveled distances classified based on group and directions. In each plot, the regression line for the Follower and Leader are indicated. The upper regression equation is for the Leader and the lower is for the Follower. 
A similar relationship between movement time and traveled distances were observed for both subjects in the same group with the same direction cooperative task. Nevertheless, the relationship for both subjects in the same group differs when the task was executed in a different direction. In forward/backward task the similarity of the relationship between subjects (same direction) existed because the object could be moved easily in one dimensional motion (a straight line with a single coordinate movement). Thus, both subjects could start and stop the cooperative task simultaneously. Most cases show that the forward direction generated a shorter movement time than the backward task. However, the statistical test performed (same group and same subject) indicated that the effect of task direction on the movement time was not significant, all $p \mathrm{~s}<0.01$.
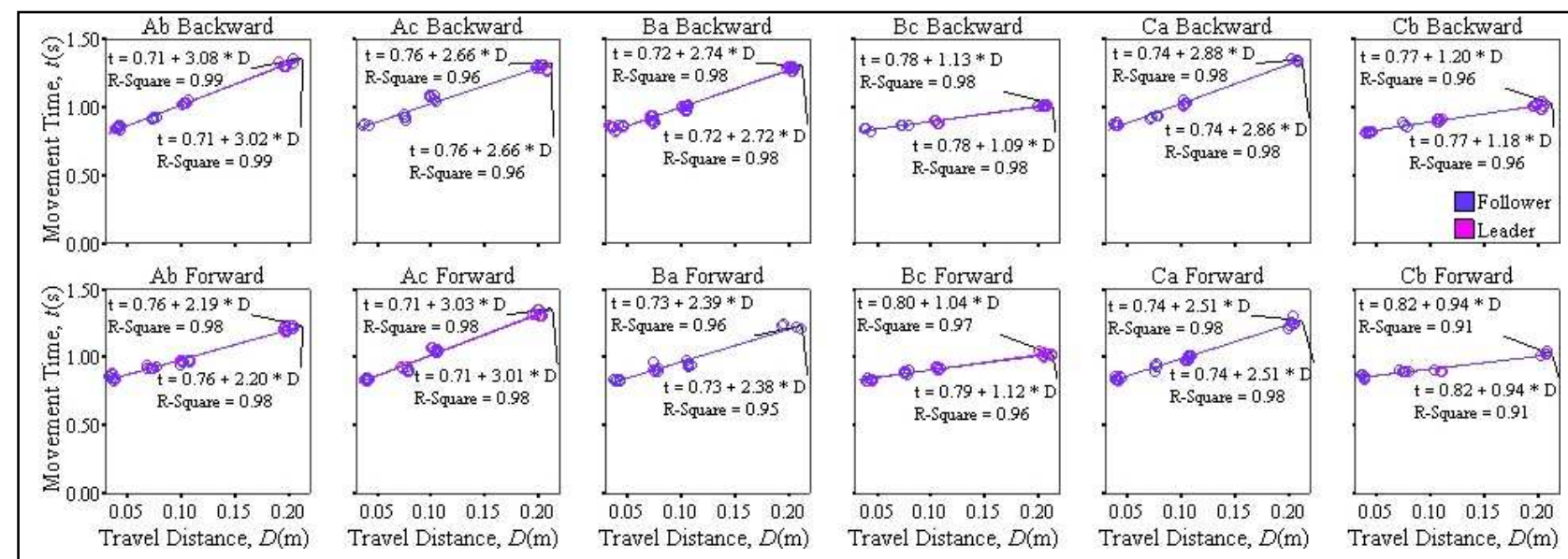

Fig. 5 Cooperative task movement time and traveled distances classified based on group and direction

\section{Conclusion}

The research investigated the relationship between movement time and traveled distances in a cooperative task performed by two humans (known as Leader and Follower) in forward/backward direction. The relationship was required by the Minimum Jerk Model to generate a smooth manipulator motion in human-robot system mimicking the smoothness of human Follower hand motion in human-human system. The results indicated that the movement time was linearly related to the traveled distances. Both Leader and Follower showed a similar relationship for the task executed in the same direction. In forward/backward cooperative task, the direction was found not significant in influencing the relationship between movement time and traveled distances. In the next phase of the research, the above relationship will be tested in human-robot system.

\section{References}

[1] Glasauer S., Huber M., Basili P., Knoll A., and Brandt T., Interacting in time and space: Investigating human-human and human-robot joint action, Proceeding of IEEE international symposium on robot and human interactive communication, Italy, 2010, 252-257.

[2] Yusuke Maeda, Takayuki Hara, Tamio Arai, Human-robot cooperative manipulation with motion estimation, Proceeding of IEEE/RSJ international conference on intelligent robots and systems,USA,2001, 2240-2245.

[3] Shahriman Abu Bakar, Ryojun Ikeura, Yuichiro Handa, Takemi Yano, Kazuki Mizutani, Hideki Sawai, Communication during the Cooperative Motion in the Task of carrying an object between two humans, Journal of Biomechanical Science and Engineering, Vol. 5, No.2(2010), 104-118.

[4] T. Flash, N. Hogan, The coordination of the arm movement: an experimentally confirmed mathematical model, Journal of neuroscience, Vol.5, No. 7(1985), 1688-1703.

[5] Brach Poston, Arend W.A. Van Gemmert *, Beth Barduson, George E. Stelmach, Movement structure in young and elderly adults during goal-directed movements of the left and right arm, Brain \& Cognition, 69 (2009) 30-38. 